\title{
The Use of Immersive Virtual Reality for Vocabulary Acquisition: a Systematic Literature Review
}

\author{
Eduardo G. Q. Palmeira, Victor B. S. Martin, Victor B. Gonçalves, \\ Ígor A. Moraes, Edgard A. Lamounier Jr., Alexandre Cardoso \\ Faculdade de Engenharia Elétrica - Universidade Federal de Uberlândia (UFU) \\ Uberlândia - MG - Brazil \\ \{egqpalmeira, victor.biagiotti, barbosagoncalves.victor, \\ igorandradesystem\}@gmail.com, \{lamounier, alexandre\}@ufu.br
}

\begin{abstract}
Many teachers consider vocabulary acquisition to be a low intellectual activity. However, this subject demands substantial time from both teachers and students, since there is a vast number of words to be learned. In this context, one expects that vocabulary acquisition assisted by Immersive Virtual Reality (IVR) could potentially be used as a powerful learning method. Therefore, this paper summarizes the state-of-the-art concerning the use of IVR in vocabulary acquisition through a systematic literature review. The obtained results showed not only that IVR facilitates autonomous learning, but that it can also affect the educational process, pointed out by an increase in its speed, higher effectiveness, positive attitudes, engagement, and motivation on the part of students.
\end{abstract}

\section{Introduction}

Computer-Assisted Language Learning (CALL) is a technique focused at Foreign Language (FL) acquisition through the development of new technologies and approaches [Stockwell 2012]. Distractions are usually common during FL acquisition; therefore, technologies and approaches that immerse FL learners in a learning environment, isolated from the real world, have more potential to ensure focused students [Chapelle and Jamieson 1986]. Appropriately, support for CALL can be found by using various immersive technologies, such as Virtual Reality (VR) [Schwienhorst 2002].

VR is the immersion of real individuals in virtual worlds created by computers. This immersion is generated through human interaction with virtual objects and their manipulation, creating in the users the feeling that they are part of the virtual world [Magnenat-Thalmann and Thalmann 1994]. Thus, by means of VR applications, the simulation of real-world situations is possible through interactive computer-generated 3D environments for FL learning purposes. In particular, in Immersive Virtual Reality (IVR), technological devices, such as Head-Mounted Display (HMD), are used to provide greater immersion to the user.

The use of HMD in the educational context allows the learner to get immersed into challenging and educational situations, also allowing them to practice the lesson as many times as needed or desired [Jensen and Konradsen 2018]. Moreover, superior retention and recall memory skills are some of the benefits of IVR through HMD compared to the traditional VR desktop condition [Krokos et al. 2019]. In other words, IVR creates more memorable experiences and plays a significant role in helping to recall learned information. In addition, its use has shown to be related to more substantial learning and performance [Fonseca and Otsuka 2017]. 
The real-life full immersion in the natural language environment is the most effective method for learning a FL [Kaplan-Rakowski and Wojdyński 2018, Terehoff 2000]. However, due to the lack of resources, for example, real-life immersion at countries that have its native language as the desired one for the learner is not always a viable or accessible option [Freed 1998]. Thus, IVR is an alternative method that opens the possibility for the immersion of the FL learner in life-like simulated virtual environments without the need to physically dislocate [Legault et al. 2019].

Language learning covers several main topics, but this research has its focus only on vocabulary acquisition. Many teachers consider vocabulary acquisition to be a low intellectual activity, and as such assume that students are able to learn by themselves. Therefore, teachers usually use class time for other subjects (e.g., grammar) [Coady 1996]. However, according to [Nation 2001], a substantial time is needed for teachers and learners in vocabulary acquisition, since there is a vast number of words to be learned. Moreover, this subject involves active memorization. Therefore, applications that can enhance the effectiveness of this activity and motivate the learner are essential. In this context, one expects that through immersion, visualization, navigation, and interaction with the virtual environment, the acquisition of FL vocabulary assisted by IVR, could potentially be used as a powerful learning method.

In this regard, other Systematic Literature Review (SLR) correlated with the use of VR for language learning was identified in the literature. Unlike the present review, which searched for papers published until November 2019, [Lin and Lan 2015] assessed papers from 2004 to 2013. Also, the main focus of the [Lin and Lan 2015] review was the qualitative analysis of papers concerning language learning in the big picture, that being, analyzing VR tendencies. On the other hand, the present SLR presents a stateof-the-art update, which focuses only on vocabulary acquisition by the exclusive use of IVR. Therefore, this paper summarizes the state-of-the-art concerning the use of IVR in vocabulary acquisition through a review of the existing literature, in order to identify gaps that future investigations can address.

\section{Methodology}

This paper presents a SLR [Kitchenham and Charters 2007]. This type of research is a secondary study conducted by a well-defined methodology, which aims to identify, analyze and interpret relevant primary study results concerning a particular topic. In addition to identifying the state-of-the-art of a research topic, SLR should reduce bias, thus distinguishing itself from the traditional literature review. In this way, three Research Questions (RQs) were defined in order to determine which information would be searched within the studies included in the qualitative synthesis. In such, the answers of the RQs should point out relevant topics and limitations from the current state-of-the-art. Hence, this SLR aimed to answer the following RQs: (1) What are the advantages and disadvantages of using IVR in vocabulary acquisition settings? (2) What are the main findings in this research area? (3) Which strategies and vocabularies are chosen to explore the exclusive benefits from the use of IVR technology?

As seen in Figure 1, this SLR's first step was to identify records regarding the research topic. Then, the filtering of the study was performed in two phases: screening and eligibility. Accordingly, at the end of the selection process, relevant studies were 
included for the review. Such steps will be given a detailed description in proceeding areas of this study.

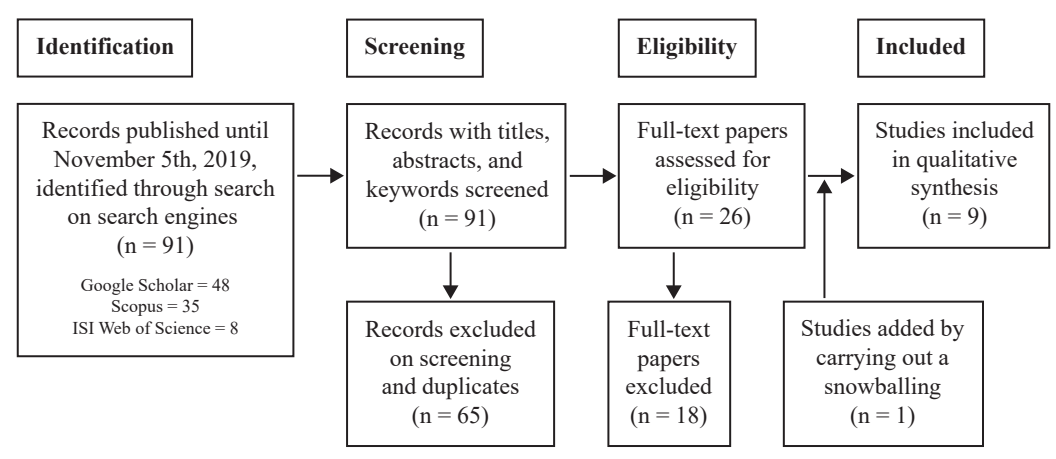

Figure 1. Selection process of the studies included in this SLR

The identification step is performed through the use of the inclusion criteria and search strings. Inclusion criteria are minimum requirements that determine which records will be taken to the selection phases. For this review, the inclusion criteria were studies published until November 5th, 2019, that addressed VR applications for language learning, as disposed on Table 1.

Table 1. Inclusion Criteria (IC), Exclusion Criteria (EC) and their descriptions

\begin{tabular}{|l|l|l|}
\hline Criteria & ID & Description \\
\hline Inclusion & IC.1 & Papers published until November 5th, 2019 \\
\cline { 2 - 3 } & IC.2 & Studies that addressed VR applications for language learning \\
\hline \multirow{4}{*}{ Exclusion } & $\boldsymbol{E C} .1$ & $\begin{array}{l}\text { Studies that addressed VR applications for language learning, but was not focused on vocabulary } \\
\text { acquisition }\end{array}$ \\
\cline { 2 - 3 } & $\boldsymbol{E C} .2$ & Studies that did not address IVR applications \\
\cline { 2 - 3 } & $\boldsymbol{E C} .3$ & Studies that are books, abstracts, theses or dissertations \\
\cline { 2 - 3 } & $\boldsymbol{E C} .4$ & Non-primary studies \\
\cline { 2 - 3 } & $\mathbf{E C . 5}$ & Papers written in a language other than English \\
\hline
\end{tabular}

Search strings are composed by relevant keywords concerning the research topic. Through the use of these strings, advanced searches are performed on databases and search engines. A well-defined search string ensures the inclusion of relevant records to the future process of paper selection. In terms of the study herein, while aiming at a wider range of studies, the search was performed on the main search engines Scopus, ISI Web of Science, and Google Scholar. For Scopus and ISI Web of Science, the search string was applied to the titles, abstracts, and keywords of each paper. Hereafter, the following search string was used: (("virtual reality" OR vr OR "immersive virtual environment”) AND language AND (acquisition OR learning OR teaching) AND vocabulary). Alternatively, for Google Scholar, the following string was applied only to titles: ("virtual reality" AND language AND (learning OR acquisition)).

This adaptation is justified by the differences in performing an advanced search in Google Scholar. Such a search engine only has options for searching the string based on either the full-text or just on the title. However, a search based on the full-text resulted in thousands of papers, many with little relevance and containing only mentions of the 
keywords in the theoretical background, which leads to a difficult selection process. Subsequently, it was decided to perform an advanced search based only on the titles of the studies. Therefore, since fetching keywords based only on the title restricts some relevant results, the search string was adapted with less keywords in order to become more comprehensive and inclusive, so as to try to mitigate this difference. Consequently, even though the adapted search string has identified some records related to FL learning, but not so related to vocabulary acquisition, this different strategy for this specific search engine proved to be more efficient, resulting in more relevant studies on the research topic than the search based on the full-text.

In the first step, 91 records were identified, 48 from Google Scholar, 35 from Scopus, and 8 from ISI Web of Science (Figure 1). Subsequently, by starting out from the screening phase, the selection steps were initiated. At this point, the exclusion criteria (Table 1) guided the filtering process of the records. Hence, after the initial 91 records were screened, based on titles, abstracts, and keywords, 26 records remained. The other 65 records were excluded through the exclusion criteria or because they were duplicates.

In the eligibility step, the exclusion criteria were also used as references, although based on the full-text of the papers. Moreover, in order to perform a more accurate assessment, papers were included if at least one RQ had been answered. Therefore, at the end of the eligibility step, 18 papers were excluded from among the 26 assessed, which resulted in 8 selected papers. Following this, in order to ensure that relevant studies were not wasted, at the end of the study selection, a backward snowballing ${ }^{1}$ was carried out based on the references of the selected papers, which went on to add 1 new paper. Finally, 9 studies were included as the most relevant when it came to answering the RQs. These can be seen on Table 2 .

Table 2. List of included studies and $R Q$ answered by these.

\begin{tabular}{|c|c|c|}
\hline Reference & Title & RQ Answered \\
\hline $\begin{array}{l}\text { [Repetto et al. } \\
\text { 2015] }\end{array}$ & $\begin{array}{c}\text { Is Motor Simulation Involved During Foreign Language Learning? A Virtual Reality } \\
\text { Experiment }\end{array}$ & RQ2 and RQ3 \\
\hline $\begin{array}{l}\text { [Ebert et al. } \\
\text { 2016] }\end{array}$ & A Virtual Reality Language Acquisition System & $\begin{array}{l}\text { RQ1, RQ2 and } \\
\text { RQ3 }\end{array}$ \\
\hline $\begin{array}{l}\text { [Cheng et al. } \\
\text { 2017] }\end{array}$ & Teaching Language and Culture with a Virtual Reality Game & $\begin{array}{l}\text { RQ1, RQ2 and } \\
\text { RQ3 }\end{array}$ \\
\hline [Xu et al. 2017] & $\begin{array}{l}\text { The Design and Implementation of Chinese Vocabulary Learning Case Based on Mobile } \\
\text { VR for "The Belt and Road" }\end{array}$ & RQ2 \\
\hline $\begin{array}{l}\text { [Dobrova et al. } \\
\text { 2018] }\end{array}$ & Virtual and Augmented Reality in Language Acquisition & $\begin{array}{l}\text { RQ1, RQ2 and } \\
\text { RQ3 }\end{array}$ \\
\hline $\begin{array}{l}\text { [Rakowski and } \\
\text { Wojdynski 2018] }\end{array}$ & Students' Attitudes Toward High-Immersion Virtual Reality Assisted Language Learning & $\begin{array}{l}\text { RQ1, RQ2 and } \\
\text { RQ3 }\end{array}$ \\
\hline $\begin{array}{l}\text { [Vázquez et al. } \\
\text { 2018] }\end{array}$ & Words in Motion: Kinesthetic Language Learning in Virtual Reality & $\begin{array}{l}\text { RQ1, RQ2 and } \\
\text { RQ3 }\end{array}$ \\
\hline $\begin{array}{l}\text { [Legault et al. } \\
\text { 2019] }\end{array}$ & $\begin{array}{c}\text { Immersive Virtual Reality as an Effective Tool for Second Language Vocabulary } \\
\text { Learning }\end{array}$ & $\begin{array}{l}\text { RQ1, RQ2 and } \\
\text { RQ3 }\end{array}$ \\
\hline [Xie et al. 2019] & Effects of Using Mobile-Based Virtual Reality on Chinese L2 Students' Oral Proficiency & RQ1 and RQ2 \\
\hline
\end{tabular}

\footnotetext{
${ }^{1}$ In this process, the list of references of the selected studies is analyzed in an attempt to identify additional relevant studies that were not found by applying the search string [Wohlin 2014]
} 
IX Congresso Brasileiro de Informática na Educação (CBIE 2020)

Anais do XXXI Simpósio Brasileiro de Informática na Educação (SBIE 2020)

\section{Results}

Data extraction was performed across all included studies $(n=9)$ from a complete reading. Accordingly, a comprehensive qualitative synthesis of each included study was made.

\section{RQ1 - What are the Advantages and Disadvantages of Using IVR in Vocabulary Acquisition Settings?}

In many studies, a comparison was made between the use of IVR and traditional methods for vocabulary acquisition in language learning settings. Here are presented a few examples of the traditional methods identified in included studies: memorization using flashcards [Ebert et al. 2016], word-word paired association learning [Legault et al. 2019], and text-only condition learning [Vázquez et al. 2018]. The use of IVR has shown to benefit more the less successful learners when compared to traditional methods [Legault et al. 2019]. In other words, the IVR application has shown to have the potential to be more efficient for low-performance learners.

In regards to user preferences, the ratings and enjoyment perceived through IVR, when compared to traditional methods, were more significant [Ebert et al. 2016]. Despite this, immediately after using traditional methods, higher memory retention was reported [Ebert et al. 2016, Vázquez et al. 2018]. However, with the use of IVR, there was a higher retention of long-term memory [Ebert et al. 2016]. This consequently proves a greater effectiveness when using IVR, since the goal of language learning is the vocabulary retention to long-term memory. In addition, the use of IVR improved student oral proficiency, enriching their presentations in content and vocabulary when compared to a method that does not incorporate VR tools [Xie et al. 2019].

The reason behind the traditional methods being initially more effective can be explained by the distraction caused by learner adaptation regarding the use of VR technology [Ebert et al. 2016, Vázquez et al. 2018, Cheng et al. 2017]. This may be a characteristic of the experience generated by IVR as a new tool for language learning [Vázquez et al. 2018]. Noteworthy also is that traditional methods are familiar and used in a more natural way [Ebert et al. 2016], while IVR may be less preferred due to the complex usability perceived by some students [Legault et al. 2019].

Notwithstanding, the use of IVR generated high engagement, focus, precision, immersion, involvement, and positive attitudes, in addition to isolating users from real-world distractions [Legault et al. 2019, Cheng et al. 2017, Kaplan-Rakowski and Wojdyński 2018]. Other advantages mentioned were the improvement in the quality and speed of the educational process, as it consumes less time and uses fewer teachers during the FL learning process [Dobrova et al. 2018].

Finally, some of the reported disadvantages of using the IVR were the discomfort caused by cables and the HMD [Ebert et al. 2016]. Cybersickness, dizziness, headache, tired eyes, and poor image quality were other negative points of using IVR, mentioned by some users [Ebert et al. 2016, Cheng et al. 2017, Kaplan-Rakowski and Wojdyński 2018]. However, specially due to technology advancement, it is very likely that these limitations are soon to be overcomed, which potentially leads to the provision of advantages even greater than those currently experienced by FL learners. 
IX Congresso Brasileiro de Informática na Educação (CBIE 2020)

Anais do XXXI Simpósio Brasileiro de Informática na Educação (SBIE 2020)

\section{RQ2 - What are the Main Findings in This Research Area?}

In the study by [Ebert et al. 2016], actions taken toward learning vocabulary were not used. Alternatively, [Repetto et al. 2015] used actions in the study, but did not use specific combinations between user movements and the semantics of vocabularies. However, in the [Vázquez et al. 2018] study, IVR, with the use of kinesthetic movements, has shown to make the experience more memorable, besides helping the participants to retain a higher number of words when compared to not using such movements. Therefore, the kinesthetic component, creating a direct association between action and language, plays an essential role in vocabulary retention.

Cognitive processes, such as a sense of presence, have proven capable of both interfering and promoting the virtual experience [Repetto et al. 2015]. In the aforementioned study, there was a clear understanding that the more users perceive their actions interacting with the virtual world, the more they feel immersed and engaged. Therefore, such experiences influence their perceived sense of presence. Hence, it is essential to understand clearly how presence affects the language learning process.

Furthermore, the use of IVR was identified as being able to improve the sense of cultural involvement of the learner [Cheng et al. 2017]. The interaction and immersion of IVR are also factors that make it a very effective tool for FL learning, as it assimilates real-life experiences. By allowing the visualization of virtual objects, the possibility of generating higher vocabulary retention is conceivable [Ebert et al. 2016]. However, when comparing contexts with more navigation in the virtual environment and more interaction with virtual objects, the one with more interaction brought more accurate learning, possibly due to the manipulation of virtual objects. Accordingly, virtual environments with high immersion and interactivity contribute to an embedded and effective learning experience [Legault et al. 2019].

Different visualization and interaction devices were used in IVR applications. For visualization, the following were used: HMDs connected to a high-performance computer or notebook [Ebert et al. 2016, Legault et al. 2019, Vázquez et al. 2018, Cheng et al. 2017, Dobrova et al. 2018, Repetto et al. 2015], portable VR headset [Kaplan-Rakowski and Wojdyński 2018], and mobile-based VR [Xie et al. 2019, Xu et al. 2017]. HMDs, such as the Oculus Rift ${ }^{2}$ and HTC Vive ${ }^{3}$ offer higher image quality. However, besides these being the most expensive devices, they also depend on a high-performance computer in order to process the images that will be displayed on these visual output devices. The portable headset, Oculus $\mathrm{GO}^{4}$, does not depend on a computer and is cheaper, but runs applications with limited interaction, processing, and, as such, delivers limited image quality. Finally, despite being the most limited, mobile-based VR are more accessible to the public, as these are low-cost VR devices. A learner only depends on a smartphone compatible with VR technology, and a "box" such as Google Cardboard", to place on the learner's face into which the smartphone is inserted, in order to be used as a display.

In terms of interaction, a variety of devices were used, such as a

\footnotetext{
${ }^{2}$ https://www.oculus.com/rift

${ }^{3}$ https://www.vive.com/us

${ }^{4}$ https://www.oculus.com/go

${ }^{5}$ https://arvr.google.com/cardboard
} 
joypad [Repetto et al. 2015], Myo armband ${ }^{6}$ [Ebert et al. 2016], hand-held controllers [Legault et al. 2019, Vázquez et al. 2018], Leap Motion tracking device ${ }^{7}$ [Dobrova et al. 2018], VR mouse [Kaplan-Rakowski and Wojdyński 2018], and even the conventional computer keyboard and mouse (problems reported) [Cheng et al. 2017]. This shows that the choice of devices for interaction is substantial and depends on the objective, its limitations, and types of possible interactions on the IVR application. Finally, [Cheng et al. 2017] suggest that voice recognition, as a primary input mechanism, can be a strategy for increasing the learner sense of presence, while at the same time reducing the dependency on the interface and the confusion of interaction that may be generated by the IVR interface.

\section{RQ3 - Which Strategies and Vocabularies are Chosen to Explore the Exclusive Benefits from the Use of IVR Technology?}

Due to the possibility for immersive interactions and the elaboration of contexts focused on learning, the selection of the addressed content in an IVR solution should be a process that is aligned with the technology in use. Therefore, the type of vocabulary that will be taught should be selected in order to maximize the learning process. Consequently, the vocabularies used in this SLR's included studies that involved grammatical classes, such as nouns and verbs [Ebert et al. 2016, Legault et al. 2019, Vázquez et al. 2018, Repetto et al. 2015]. On the subject of the nouns, the choice of the related virtual environment theme and objects aimed only at exploring the research topic of each study strategically, in order to explore the potential and particularity of IVR as a learning tool for FL. Furthermore, words that are categorized as virtual objects and can be manipulated were perceived as those that could be learned more effectively than, for example, animals in a zoo that can only be observed [Legault et al. 2019]. In relation to the used verbs, there came the realization that abstract verbs were more difficult to remember than concrete action verbs [Repetto et al. 2015].

Other studies proposed different strategies. These approaches have not focused on isolated vocabularies, but on multiple-choice conversation dialogues, challenging the learner to select an appropriate answer [Cheng et al. 2017, Kaplan-Rakowski and Wojdyński 2018]. In others, the student did not choose an entire sentence, but a part of a dialogue that was intentionally incomplete, requiring the user to select the right words [Dobrova et al. 2018] or to organize components into the correct order to construct a sentence [Cheng et al. 2017]. In conclusion, not only the type of vocabulary should be assessed, but also the learning strategies used in the applications to maximize the IVR technology benefits in vocabulary acquisition settings.

\section{Threats to Validity}

Although guarantees cannot be given that the defined RQs answer all the questions related to the research investigation - and it may happen that some have not been addressed the importance of their proper elaboration was considered. Therefore, several meetings were held with the research team to discuss and calibrate SLR RQs. In regards to the quantity of primary studies, the search string did not return a large number of results.

\footnotetext{
${ }^{6}$ https://support.getmyo.com/hc/en-us

${ }^{7}$ https://www.ultraleap.com/product/leap-motion-controller
} 
This is due to the fact that the use of IVR in CALL settings has not been widely studied as of yet. Furthermore, three main known search engines were used: Scopus, ISI Web of Science, and Google Scholar. It is worth mentioning once again that adaptations were made to the search string: in the Google Scholar search engine, due to its particularity, in order to mitigate differences and find relevant results. In addition, in order to mitigate the chances that relevant articles were not included in this SLR, a backward snowballing has been adopted. Therefore, a satisfactory number of primary studies relevant to the extraction of the necessary data to answer the RQs was found. Finally, concerning the subjectivity in data extraction, the judgments made by the SLR authors were used as the basis for the classification and data extraction of the included primary studies. For this reason, to reduce the biases of this threat, the extraction process was performed by reviewing all researchers.

\section{Conclusions}

In the included studies, the adopted vocabularies varied widely, covering topics such as physical objects to verbs describing actions. It was also noted that kinesthetic components play a significant role in word retention, as they tend to be more easily remembered. In addition, studies point out that IVR enables autonomous learning with an increase in the speed of the educational process, thus reducing costs and the number of dedicated teachers needed to this field.

Moreover, differences in vocabulary acquisition using IVR and traditional methods were identified through the processes conducted in this study. Traditional methods produce higher short-term memory retention when compared with using IVR, due for example, to the initial distraction caused by the VR system. Further, it is noteworthy the existence of a relationship between the interaction level of the user and accurate learning. Similarly, according to the [Fonseca and Otsuka 2017]'s review, natural interactions by means of gestures present superior results when compared to non-natural interactions performed by using joystick or keyboard and mouse. However, natural interaction may be harder to master, as this type of interaction can distract the user. In contrast, nonnatural interaction is more appropriate in activities that demand accuracy and lower time for training. In short, the analyzed results for this SLR have shown that over the longterm IVR produces higher word retention to the language learner, due to the possibility of visualization and manipulation of virtual objects.

Notably, according to [Ma and Kelly 2006], a common feature used in CALL programs are in-context vocabulary learning. Following on with this point of view, [Schwienhorst 2002] noted a more significant cognitive involvement when the student was immersed in a user-centered virtual scenario and also able to control this environment. Suitably, such mentioned features are possibilities that can be found in IVR applications for vocabulary acquisition. Furthermore, by assessing the studies included in this SLR, the use of IVR has shown higher effectiveness, positive attitudes, engagement, and motivation on the part of students. Also, it is noteworthy that IVR usage has shown to provide more positive results for students with lower performance on the knowledge assessment of the studies than traditional approaches.

Although the synthesized results for this SLR were positive, the low number of included studies for this review $(n=9)$ can indicate that further investigations are neces- 
sary. Besides, some topics were identified as gaps for future research: (1) How the negative impacts and limitations using IVR for vocabulary acquisition could impair learning? (2) How sense of presence on the part of the user affects the vocabulary acquisition process? (3) Which strategies that aim to balance the performance and accessibility of IVR devices could be incentivized to increase the technology implementation in vocabulary acquisition settings? (4) How could interaction by voice recognition usage in IVR applications increase vocabulary retention? (5) How could learning be boosted through levels of interaction, spatial navigation, and immersion regarding IVR usage for vocabulary acquisition?

\section{Acknowledgment}

This study was financed in part by the Coordenação de Aperfeiçoamento de Pessoal de Nível Superior - Brasil (CAPES) - Finance Code 001.

\section{References}

Chapelle, C. and Jamieson, J. (1986). Computer-assisted language learning as a predictor of success in acquiring english as a second language. TESOL quarterly, 20(1):27-46.

Cheng, A., Yang, L., and Andersen, E. (2017). Teaching language and culture with a virtual reality game. In Proceedings of the 2017 CHI Conference on Human Factors in Computing Systems, CHI '17, page 541-549, New York, NY, USA. Association for Computing Machinery.

Coady, J. (1996). L2 vocabulary acquisition: A synthesis of the research, pages 273290. Cambridge Applied Linguistics. Cambridge University Press.

Dobrova, V., Labzina, P., Ageenko, N., Nurtdinova, L., and Elizarova, E. (2018). Virtual and augmented reality in language acquisition. In Proceedings of the International Conference on the Theory and Practice of Personality Formation in Modern Society (ICTPPFMS 2018), pages 218-223. Atlantis Press.

Ebert, D., Gupta, S., and Makedon, F. (2016). Ogma: A virtual reality language acquisition system. In Proceedings of the 9th ACM International Conference on PErvasive Technologies Related to Assistive Environments, PETRA '16, New York, NY, USA. Association for Computing Machinery.

Fonseca, L. and Otsuka, J. (2017). Aplicações educacionais com óculos de realidade virtual: um mapeamento sistemático. Brazilian Symposium on Computers in Education (Simpósio Brasileiro de Informática na Educação - SBIE), 28(1):233-242.

Freed, B. F. (1998). An overview of issues and research in language learning in a study abroad setting. Frontiers: The Interdisciplinary Journal of Study Abroad, 4(1):31-60.

Jensen, L. and Konradsen, F. (2018). A review of the use of virtual reality headmounted displays in education and training. Education and Information Technologies, 23(4):1515-1529.

Kaplan-Rakowski, R. and Wojdyński, T. (2018). Students' attitudes toward highimmersion virtual reality assisted language learning. In Taalas, P., Jalkanen, J., Bradley, L., and Thouësny, S., editors, Future-proof CALL: language learning as exploration and encounters-short papers from EUROCALL 2018, pages 124-129. Research-publishing.net, Voillans. 
IX Congresso Brasileiro de Informática na Educação (CBIE 2020)

Anais do XXXI Simpósio Brasileiro de Informática na Educação (SBIE 2020)

Kitchenham, B. and Charters, S. (2007). Guidelines for performing systematic literature reviews in software engineering. Technical Report EBSE-2007-01, 2.

Krokos, E., Plaisant, C., and Varshney, A. (2019). Virtual memory palaces: Immersion aids recall. Virtual Reality, 23(1):1-15.

Legault, J., Zhao, J., Chi, Y.-A., Chen, W., Klippel, A., and Li, P. (2019). Immersive virtual reality as an effective tool for second language vocabulary learning. Languages, 4(1):13.

Lin, T.-J. and Lan, Y.-J. (2015). Language learning in virtual reality environments: Past, present, and future. Journal of Educational Technology \& Society, 18(4):486-497.

Ma, Q. and Kelly, P. (2006). Computer assisted vocabulary learning: Design and evaluation. Computer Assisted Language Learning, 19(1):15-45.

Magnenat-Thalmann, N. and Thalmann, D. (1994). Artificial Life and Virtual Reality. Wiley.

Nation, I. S. P. (2001). Learning Vocabulary in Another Language. Cambridge Applied Linguistics. Cambridge University Press.

Repetto, C., Colombo, B., and Riva, G. (2015). Is motor simulation involved during foreign language learning? a virtual reality experiment. SAGE Open, 5(4):1-10.

Schwienhorst, K. (2002). Why virtual, why environments? implementing virtual reality concepts in computer-assisted language learning. Simulation \& Gaming, 33(2):196209.

Stockwell, G. (2012). Computer-Assisted language learning: Diversity in research and practice. Cambridge University Press.

Terehoff, I. (2000). Learning by living the language: The benefits of foreign exchange programs. National Association of Secondary School Principals. NASSP Bulletin, 84(612):83.

Vázquez, C., Xia, L., Aikawa, T., and Maes, P. (2018). Words in motion: Kinesthetic language learning in virtual reality. In 2018 IEEE 18th International Conference on Advanced Learning Technologies (ICALT), pages 272-276.

Wohlin, C. (2014). Guidelines for snowballing in systematic literature studies and a replication in software engineering. In Proceedings of the 18th International Conference on Evaluation and Assessment in Software Engineering, EASE '14, New York, NY, USA. Association for Computing Machinery.

Xie, Y., Chen, Y., and Ryder, L. H. (2019). Effects of using mobile-based virtual reality on chinese 12 students' oral proficiency. Computer Assisted Language Learning, pages $1-21$.

Xu, Y., Zheng, S., Chen, Q., Ou, S., Liu, C., and Xiao, Y. (2017). The design and implementation of chinese vocabulary learning case based on mobile vr for "the belt and road". In International Conference on Computational Modeling, Simulation and Applied Mathematics, CMSAM 2017, pages 263-266. DEStech Transactions on Computer Science and Engineering. 